Research Paper

\title{
The Effects of Naturalistic Light on Diurnal Plasma Melatonin and Serum Cortisol Levels in Stroke Patients during Admission for Rehabilitation: A Randomized Controlled Trial
}

\author{
Anders S West ${ }^{\circledR}{ }^{\bowtie}$, Henriette P Sennels ${ }^{2}$, Sofie A Simonsen ${ }^{1}$, Marie Schønsted ${ }^{1}$, Alexander H Zielinski ${ }^{1}$, \\ Niklas C Hansen ${ }^{1}$, Poul J Jennum³ ${ }^{\text {, Birgit Sander }}{ }^{4}$, Frauke Wolfram5, Helle K. Iversen ${ }^{1}$ \\ 1. Clinical Stroke Research Unit, Department of Neurology, Rigshospitalet, Faculty of Health Sciences, University of Copenhagen. \\ 2. Department of Clinical Biochemistry, Rigshospitalet and Faculty of Health Sciences, University of Copenhagen. \\ 3. Danish Center for Sleep Medicine, Department of Neurophysiology Rigshospitalet, Faculty of Health Sciences, University of Copenhagen. \\ 4. Department of Ophthalmology, Rigshospitalet, Copenhagen University Hospital. \\ 5. Department of diagnostic, Radiologic clinic, Rigshospitalet and Faculty of Health Sciences, University of Copenhagen.
}

$\square$ Corresponding author: Anders Sode West: MD, Clinical Stroke Research Unit, N25, Department of Neurology, Rigshospitalet, Glostrup, Faculty of Health Sciences, University of Copenhagen. Address: City: Copenhagen, Zip code: 2600. Road: Valdemar Hansens Vej 1-23. Mail: anders.sode.west@regionh.dk, tel. +45 21748587

(c) Ivyspring International Publisher. This is an open access article distributed under the terms of the Creative Commons Attribution (CC BY-NC) license (https://creativecommons.org/licenses/by-nc/4.0/). See http://ivyspring.com/terms for full terms and conditions.

Received: 2018.07.30; Accepted: 2018.11.29; Published: 2019.01.01

\begin{abstract}
Background: Stroke patients admitted for rehabilitation often lack sufficient daytime blue light exposure due to the absence of natural light and are often exposed to light at unnatural time points.

We hypothesized that artificial light imitating daylight, termed naturalistic light, would stabilize the circadian rhythm of plasma melatonin and serum cortisol levels among long-term hospitalized stroke patients.

Methods: A quasi-randomized controlled trial. Stroke patients in need of rehabilitation were randomized between May 1, 2014, and June 1, 2015 to either a rehabilitation unit equipped entirely with always on naturalistic lighting (IU), or to a rehabilitation unit with standard indoor lighting (CU). At both inclusion and discharge after a hospital stay of at least 2 weeks, plasma melatonin and serum cortisol levels were measured every 4 hours over a 24 -hour period. Circadian rhythm was estimated using cosinor analysis, and variance between time-points.

Results: A total of 43 were able to participate in the blood collection. Normal diurnal rhythm of melatonin was disrupted at both inclusion and discharge. In the IU group, melatonin plasma levels were increased at discharge compared to inclusion $(n=23$; median diff, $2.9 ; \mathrm{IQR}:-1.0$ to $9.9, p=0.030)$ and rhythmicity evolved $(n=23 ; p$ $=0.007$ ). In the $\mathrm{CU}$ group, melatonin plasma levels were similar between discharge and inclusion and no rhythmicity evolved. Overall, both patient groups showed normal cortisol diurnal rhythms at both inclusion and discharge.

Conclusions: This study is the first to demonstrate elevated melatonin plasma levels and evolved rhythmicity due to stimulation with naturalistic light.
\end{abstract}

Key words: stroke, rehabilitation, circadian rhythm, light, melatonin, cortisol

\section{Introduction}

Interventional uses of light have attracted growing interest since the recent discovery of the blue light absorbing Melanopsin-expressing photosensitive ganglion cells (ipRGCs) in the retinal ganglion cell layer. Especially a subtype of ipRGCs (M1) pass the highest amount of light stimulation through the optic nerve and retinohypothalamic tract to the master circadian clock system in the suprachiasmatic nucleus (SCN). Several studies indicate that sunlight is the strongest entrainment for the circadian rhythm because of the sensitivity for short-wavelength blue light [1]. Light stimulation to the SCN also happens 
through the intergeniculate leaflet (IGL), which appears to be an important secondary route for sunlight entrainment [2]. The SCN affects melatonin and cortisol in a manner involving the oscillation system within the $\mathrm{SCN}$ and its direct autonomic connection with peripheral tissue.

Melatonin is produced from serotonin in the pineal gland, and its circuitous pathway is regulated by the SCN. Light normally inhibits melatonin secretion, such that it is low during the day and peaks late at night, and this temporal pattern is relatively unaltered by changes in sleep habits [3]. During hospitalization, critically ill patients reportedly exhibit low melatonin levels and a disrupted diurnal melatonin rhythm $[4,5]$. Patients with cortical stroke also show decreased melatonin secretion [6-8] and a disturbed diurnal rhythm [9]. Although the physiological explanation of this phenomenon is unknown. It is possible that the initial edema and widespread cortical lesions may affect areas projecting to the IGL, impairing light perception to the SCN, and through that disrupting circadian rhythm regulation [6].

Another well-known circadian-regulating hormone, cortisol, synchronizes peripheral circadian oscillators and controls $60 \%$ of the circadian transcriptome [10]. Cortisol secretion is controlled by the $\mathrm{SCN}$, where neuronal projections signal directly to the paraventricular hypothalamic nucleus (PVH) and dorsomedial hypothalamus (DMH). Cortisol levels normally rise around midnight, peak in the early morning, and decrease again around 9 a.m. Cortisol is reportedly elevated in response to external stimulus, such as hospital admission and surgery [11,12]. However, it seems likely that cortisol is more stable than melatonin in critically ill patients exposed to diurnal disruption [13].

Hospitalization and circadian rhythm disruption reportedly have negative consequences [14]. Patients admitted for post-stroke rehabilitation carry a high risk of circadian disruption due to the duration of hospitalization and immobilization. This combination deprives patients of natural light from the sun, subjects them to many hours of artificial light from the evening and nighttime indoor hospital lighting.

LED (light-emitting diode) technologies support the development of artificial light with specific wavelengths. Together with computerized technology, this enables the production of lamps that can imitate the natural sunlight spectrum and rhythmtermed naturalistic light, circadian light, or dynamic lighting. Melatonin levels are influenced by light interventions [15], and several studies show that short-wave light is an isolated melatonin manipulator [16-19]. Previously tested light interventions have not detectably altered melatonin levels in patients in real-hospital settings [20,21]. However, no studies have investigated the influence of naturalistic light on melatonin levels and its diurnal rhythm.

In the present study, we aimed to determine whether naturalistic light could stabilize the circadian rhythm of melatonin and cortisol, and increase the expected low plasma melatonin levels in stroke patients admitted for rehabilitation.

\section{Materials and Methods}

\section{Study design and Participants}

This study was performed in the Stroke Rehabilitation Unit, Department of Neurology, Rigshospitalet, Copenhagen. The methods have been previously described in detail [14]. Briefly, the study included stroke patients who required over 2 weeks of in-hospital rehabilitation during the period from May $1^{\text {st }}$ of 2014 to June $1^{\text {st }}$ of 2015 . Patients were excluded if they were unable to give consent due to their awareness status, severe aphasia, or less than 2 weeks of hospitalization in the rehabilitation unit. We conducted a parallel randomized controlled trial with two arms: an intervention group admitted to a rehabilitation unit equipped with naturalistic light (IU), and a control group admitted to a rehabilitation unit with standard indoor lighting (CU). No safety precautions were necessary regarding assessments and interventions. The study was approved by the Danish scientific ethics committee (H-4-2013-114) and the Danish Data Protection Agency (2007-58-0015), and is registered at ClinicalTrials.gov (Identifier: NCT02186392).

\section{Randomization}

Randomization was performed by non-blinded stroke nurses (quasi-randomization) at the acute stroke unit (with normal standard light conditions). The nurses were not involved in the study and were simply following normal procedure regarding the relocation of patients to the two rehabilitation units.

\section{Naturalistic light intervention}

In all areas of the intervention rehabilitation unit, a 24-hour naturalistic lighting scheme was implemented using multi-colored LED-based luminaires (lamps) managed by a centralized lighting controller according to the lighting scheme (Chromaviso, Denmark). The lighting was dim in the morning (from $7 \mathrm{am}$ ), increased to reach maximum illuminance between noon and $3 \mathrm{pm}$ with strong inclusion of the blue light spectrum, and then dimmed again throughout the evening with diminishment of the blue light spectrum, ensuring no IpRGC stimulation during nighttime. The luminaires 
were located in the ceiling and at the wall behind the beds, and the naturalistic lighting scheme ran constantly throughout the inclusion period.

Due to the complexity and the need for comprehensive technical description of the light, the light intervention is presented in details in the method description paper [14] where the irradiance profiles can be found in figure $3 \mathrm{a}$ and $3 \mathrm{~b}$. The technical light description is produced in accordance with CIE TN 003 following the principles of Lucas et al. [22]. Normal ceiling luminaries were installed in the CU. They had new fluorescent tubes installed prior to the inclusion in order to uniform the light in all areas of the $\mathrm{CU}$. The technical light description regard the irradiance profiles for the IU can be found in figure $3 a$ and for $\mathrm{CU}$ in $3 \mathrm{~b}$ in West et al [14].

\section{Measurements}

All acute stroke patients underwent standard initial examinations. Additionally, the MorningnessEveningness Questionnaire (MEQ) was performed at both inclusion and discharge to determine the distribution of circadian classes. Daily life in the patient ward was best suited to morning types, such that evening-type circadian class could potentially interfere with outcome for these patients. The MEQ is validated for determining individual circadian rhythm [23], and divides patients into five types: Definitely Evening Type, Moderately Evening Type, Neither Type, Moderately Morning Type, and Definitely Morning Type. The highest scores indicate the morning type.

\section{Blood samples}

Blood samples were collected at both inclusion and discharge (hospital treatment complete/done) for measurement of melatonin and cortisol levels at 4-hour intervals, seven times over a 24-hour period: 08 a.m., noon, 04 p.m., 08 p.m., midnight, 04 a.m., and again at 08 a.m. To prevent external factors other than light from influencing plasma melatonin and serum cortisol levels, the participants were asked to avoid parameters which could influence the blood levels [14] (Table S1). Travel to different time zones and regular night work within the last 14 days were registered. The instructions were given both verbally and in writing. To avoid circadian stimulation, blood collection was performed in dim lighting from an old incandescent bulb, which has very low emission of the blue light spectrum. During collection, the lamp was pointed towards the arm, away from the patient. Blood samples were centrifuged directly after collection, and plasma and serum were separated. Samples were immediately stored at $-50^{\circ} \mathrm{C}$, and within 30 hours were stored at $-80^{\circ} \mathrm{C}$ until further analysis.

\section{Biochemical analysis}

Plasma melatonin concentrations were analyzed by use of a Melatonin Direct Radioimmunoassay (LDN Labor Diagnostika Nord GmbH and Co. Nordhorn) according to the kit instructions. The limit of detection was $2,3 \mathrm{pg} / \mathrm{mL}$, the measuring range was $2.3-1000 \mathrm{pg} / \mathrm{mL}$ and the analytical between-run coefficient of variations were $19,6 \%$ at $24 \mathrm{pg} / \mathrm{mL}$ and $14 \%$ at $70 \mathrm{pg} / \mathrm{mL}$.

Serum cortisol concentrations were determined on a Cobas e 411 analyzed (Roche Diagnostics, Basel, Switzerland) by an electro-chemiluminescence immunoassay. The limit of detection was $0.5 \mathrm{nmol} / \mathrm{L}$, the measuring range was $2-17500 \mathrm{nmol} / \mathrm{L}$ and the analytical between-run coefficient of variation was $3 \%$ at $330 \mathrm{nmol} / \mathrm{L}$.

\section{MRI radiological classification}

MRI sequences were performed, and brain lesions were classified according to volume and anatomic localization by a neuro-radiologist. The infarction volume (in $\mathrm{cm}^{3}$ ) was calculated by measuring the infarction size in the coronal, transversal, and sagittal planes. All scans were performed using a 1.5 Tesla MR scanner (Siemens, General Electrics), and included the following sequences: a sagittal T2-weighted turbo spin echo sequence (FSE), an axial T2-weighted FSE, an axial fluid attenuation inversion recovery (FLAIR) sequence, an axial 3 scan trace diffusion-weighted imaging sequence, a sagittal 3D T1WI sequence, and an axial susceptibility-weighted imaging sequence.

\section{Outcomes}

This study was part of a larger investigation of the effects of light on rehabilitation patients' health as measured by psychological parameters, biochemical parameters, fatigue, and sleep. As this subject is a relatively new scientific field, the study was considered an exploratory investigational study. We chose five primary endpoints, including melatonin and cortisol levels and rhythmicity in the present study.

\section{Statistical analysis}

All analyses were performed using SAS (SAS Inst. Inc., Cary, NC USA, 9.4). A $p$ value of $<0.05$ was considered significant. Between-group differences regarding basic demographic parameters were calculated using the $t$-test for continuous variables, and chi-square-test for categorical variables. Normally distributed continuous variables were expressed as mean \pm standard deviation (SD). The melatonin plasma levels and cortisol serum levels were not normally distributed; therefore, these data were expressed as median and interquartile range (IQR). 
Data were logarithmically transformed prior to mixed model analysis, and were subsequently transformed back to empirical fractiles to achieve parametric distribution, which were then converted to percentage variance $((x-1) * 100)$. The deviation of calculated cosinor rhythmicity was expressed as standard error (SE). Cosinor rhythmicity was analyzed assuming a 24-hour time-period [24]. The data were fitted to a combined cosine and sine function: $\mathrm{y}=\mathrm{M}+\mathrm{k} 1 \operatorname{COS}(2 \pi \mathrm{t} / 24)+\mathrm{k} 2 \operatorname{SIN}(2 \pi \mathrm{t} / 24)$. The 24-hour rhythms of each group were further characterized by the following rhythm parameters: mesor (rhythm-adjusted average about which oscillation occurs), amplitude (difference between the highest and lowest values of the fitted cosinor curve), and times of peak and nadir [24,25]. Data analyses were performed using the GPLOT procedure in SAS. Mixed model analysis was performed in SAS to describe the variance between time-points of the diurnal rhythm of melatonin and cortisol at inclusion and discharge in each unit.

Infarction size was correlated to melatonin and cortisol mean values using regression analysis. Infarction location was included as a confounding element by analysis of covariance. The Wilcoxon signed-rank test was used to describe within-group changes from inclusion to discharge. The melatonin mean plasma values were calculated from all time-points together $(24 \mathrm{~h})$. Due to the preserved diurnal rhythm, cortisol mean serum values were further divided into day (high-secretion phase; 24-12 h) and night (low-secretion phase; $12-24 \mathrm{~h}$ ) values. Melatonin plasma levels did not show a diurnal rhythm in either unit; thus, the division of mean melatonin values into further stages was not relevant.

\section{Results}

Among 256 screened patients who required in-hospital neurorehabilitation, 90 met our inclusion criteria, of whom 73 avoided meeting exclusion criteria, death, and severe illness until discharge. Of these 73 included patients, 30 dropped out before discharge, while the remaining 43 patients completed the study (Figure 1). The main reasons for missed blood collection were the patient's discomfort with the procedure, and technical complications with the first 9 included patients. Patients were also excluded from blood collection due to fragile veins and low hemoglobin concentration. Melatonin data from one patient were excluded due to prescribed melatonin treatment. Cortisol data were excluded due to very high cortisol values resulting from respiratory distress in one patient who unexpectedly died a few hours after the last blood sampling. NIHSS (Included N=43; 5.0 ( \pm 4.2$)$; excluded $\mathrm{N}=30 ; 7.8( \pm 6.4)$ : $\mathrm{p}=0.04)$ and Barthel (Included N=43; $56.9( \pm 30.0)$; excluded $\mathrm{N}=30$; 39.1 ( \pm 31.2$): p=0.02)$ scores were calculated in the group of excluded patients and indicated significant worse disability scores compared to the included participants (table S2).

A total of 33 patients were willing and able to sufficiently answer the MEQ. The two groups did not

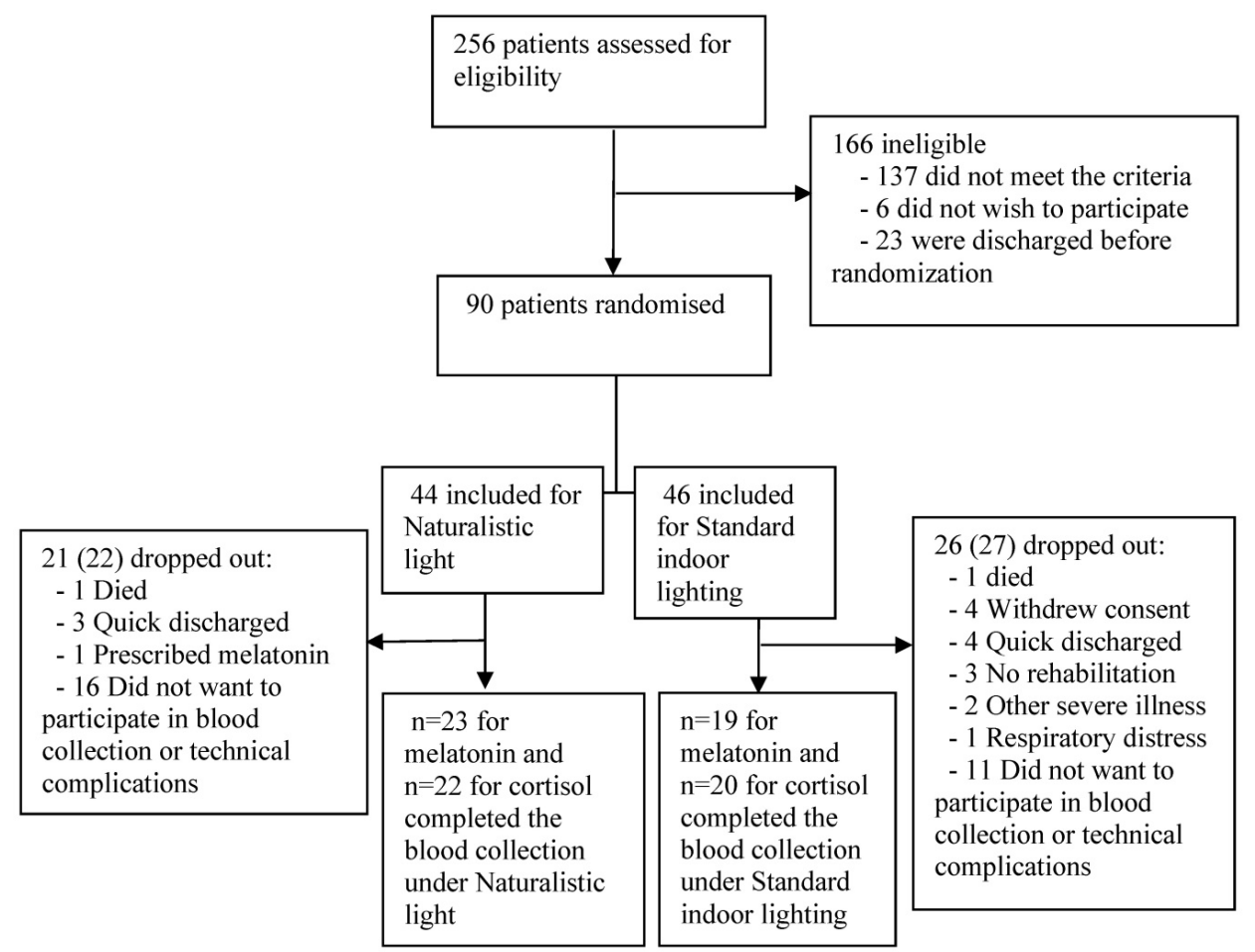

Figure 1. Trial flow chart. 
significantly differ in circadian class distribution (chisquare test) (Table 1). Table 1 presents the demographic data. The two groups were well matched, except regarding the number of smokers (IU 13, CU 16, $p=0.02$ ). Pre-analytical variability was estimated to be equal among the patients based on the information collected before blood sampling, and was therefore not included as a confounding or interaction element.

\section{Circadian rhythm of melatonin and cortisol}

At both inclusion and discharge, both patient groups lacked a normal diurnal rhythm of plasma melatonin. Melatonin plasma levels did not follow a cosinor rhythmicity in either group, at either time-point (Table 2). Regarding the variance between time-points, the $\mathrm{CU}$ group appeared to have an abnormal but diurnal melatonin rhythmicity at inclusion (Table 3). However, this rhythmicity was absent at discharge which is also illustrated by Figure 2 ,b. In the IU group at inclusion, melatonin plasma levels only significantly differed between 08 p.m. and at discharge, melatonin levels significantly differed between each time-point (Table S3), with elevated levels from 08 a.m. to noon and from 08 p.m. to midnight illustrated by Figure 2,a. In the IU group, we detected significant changes over time between inclusion and discharge. Such differences were not evident in the CU group (Table 3).

Table 1. Basic demographics of the two patient groups.

\begin{tabular}{|c|c|c|c|}
\hline Characteristic & $\begin{array}{l}\text { Intervention } \\
\text { Unit }(N=23)\end{array}$ & $\begin{array}{l}\text { Control Unit } \\
(N=20)\end{array}$ & $\begin{array}{l}p \\
\text { value }\end{array}$ \\
\hline Age, mean years (range) & $75.2(56-96)$ & $70.8(51-88)$ & 0.21 \\
\hline Sex, & & & 0.55 \\
\hline Male, $n(\%)$ & $15(65)$ & $14(74)$ & \\
\hline Female, $n(\%)$ & $8(35)$ & $5(26)$ & \\
\hline $\begin{array}{l}\text { Time from ictus to inclusion, mean days } \\
( \pm \text { SD) }\end{array}$ & $5.7( \pm 3.5)$ & $5.1( \pm 3.7)$ & 0.44 \\
\hline Admission length, mean days $( \pm S D)$ & $46.2( \pm 24.8)$ & $33.4( \pm 13.0)$ & 0.08 \\
\hline Smoker, n (\%) & $13(56.5)$ & $16(88.9)$ & 0.02 \\
\hline Hypertension, $\mathrm{n}(\%)^{*}$ & $16(70)$ & $12(63)$ & 0.66 \\
\hline \multicolumn{4}{|l|}{ Diabetes } \\
\hline Type 1, n(\%) & $0(0)$ & $2(11)$ & 0.11 \\
\hline Type 2, n(\%) & $4(17)$ & $2(11)$ & 0.53 \\
\hline Hypercholesterolemia, n (\%) & $5(22)$ & $4(21)$ & 0.96 \\
\hline Atrial fibrillation, $\mathrm{n}(\%)$ & $5(22)$ & $3(16)$ & 0.63 \\
\hline Depression, $\mathrm{n}(\%)^{* *}$ & $0(0)$ & $1(5)$ & 0.27 \\
\hline Barthel, mean score $( \pm \mathrm{SD})$ & $55.0( \pm 33.0)$ & $59.0( \pm 27.0)$ & 0.84 \\
\hline NIHSS, mean score $( \pm S D)$ & $4.9( \pm 4.1)$ & $5.2( \pm 4.5)$ & 0.94 \\
\hline MEQ total score, mean $( \pm S D)$ & $58.1( \pm 12.2)$ & $59.0( \pm 11.8)$ & 0.81 \\
\hline Definitely Evening Type, $n(\%)^{* * *}$ & $1(6.7)$ & $0(0)$ & \\
\hline Moderately Evening type, $n(\%)^{* * *}$ & $5(33.3)$ & $3(16.7)$ & \\
\hline Neither Type, $n(\%)^{* * *}$ & $2(13.3)$ & $2(11.1)$ & \\
\hline Moderately Morning Type, $n(\%)^{* * *}$ & $4(26.7)$ & $9(50.0)$ & \\
\hline Definitely Morning Type, $n(\%)^{* * *}$ & $3(20.0)$ & $4(22.2)$ & \\
\hline
\end{tabular}

Table 2. Cosinor rhythmicity of melatonin and cortisol.

\begin{tabular}{|c|c|c|c|c|c|c|c|c|}
\hline Group & $\mathbf{N}$ & Cosinor $p$ value & $\begin{array}{l}\text { Mesor (SE) } \\
(\mathrm{pg} / \mathrm{mL})\end{array}$ & ${ }^{*}$ Mesor $p$ value & Amp (SE) Peak-Nadir & $\begin{array}{l}{ }^{*} \text { Amp/ peak } p \\
\text { value }\end{array}$ & Peak time & Nadir time \\
\hline \multicolumn{9}{|l|}{ Melatonin } \\
\hline Control & & & & 0.82 & & 0.68 & & \\
\hline Inclusion & 133 & 0.07 & $23.51(1.85)$ & & $10.03(2.84)$ & & $23: 20$ & $11: 20$ \\
\hline Discharge & 130 & 0.48 & $22.54(1.66)$ & & $5.35(2,53)$ & & $23: 59$ & $11: 59$ \\
\hline Intervention & & & & 0.17 & & 0.44 & & \\
\hline Inclusion & 161 & 0.4 & $19.86(0.91)$ & & $3.30(1.38)$ & & $22: 13$ & $10: 13$ \\
\hline Discharge & 152 & 0.27 & $27.03(2.27)$ & & $9.06(3.42)$ & & $20: 23$ & $08: 23$ \\
\hline \multicolumn{9}{|l|}{ Cortisol } \\
\hline Control & & & & 0.08 & & 0.005 & & \\
\hline Inclusion & 131 & $<0.0001$ & $376.97(15.64)$ & & $270.17(24.41)$ & & $10: 48$ & $22: 48$ \\
\hline Discharged & 137 & $<0.0001$ & $311.16(12.08)$ & & $353.80(18.3)$ & & $9: 48$ & $21: 48$ \\
\hline Intervention & & & & 0.31 & & 0.84 & & \\
\hline Inclusion & 159 & $<0.0001$ & $317.48(11.45)$ & & $262.37(17.33)$ & & $10: 44$ & $22: 44$ \\
\hline Discharged & 151 & $<0.0001$ & $336.70(13.28)$ & & $286.74(20.21)$ & & $10: 33$ & $22: 33$ \\
\hline
\end{tabular}

Table 3. Calculated variance between diurnal time points for melatonin and cortisol.

\begin{tabular}{lll}
\hline Groups & p-value & p-value \\
\hline Intervention Unit & Melatonin & Cortisol \\
Inclusion & $\mathrm{NS}$ & $<.0001$ \\
Discharge & 0.002 & $<.0001$ \\
Variance, inclusion vs. discharge & 0.007 & $<.0001$ \\
Control Unit & & $<.0001$ \\
Inclusion & 0.0003 & $<.0001$ \\
Discharge & $\mathrm{NS}$ & $<.0001$ \\
Variance, inclusion vs. discharge & $\mathrm{NS}$ & \\
\hline
\end{tabular}

Type 3 tests of fixed effects was calculated based on the variance between melatonin and cortisol blood collection time-points. NS = Not significant. 
A

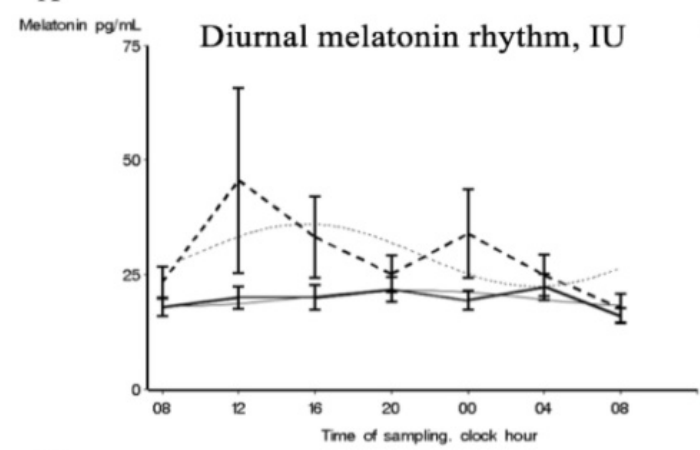

C

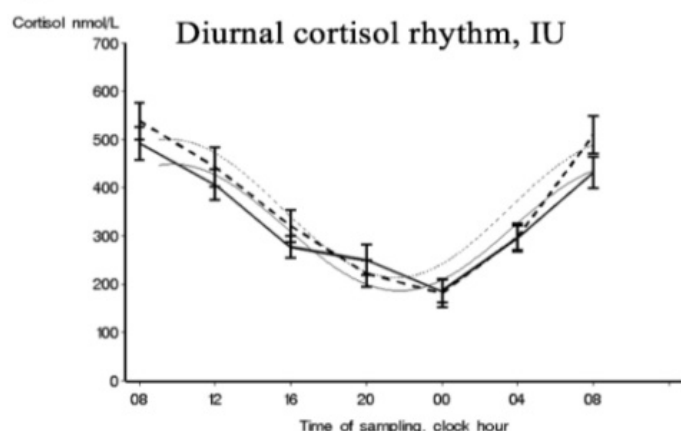

E

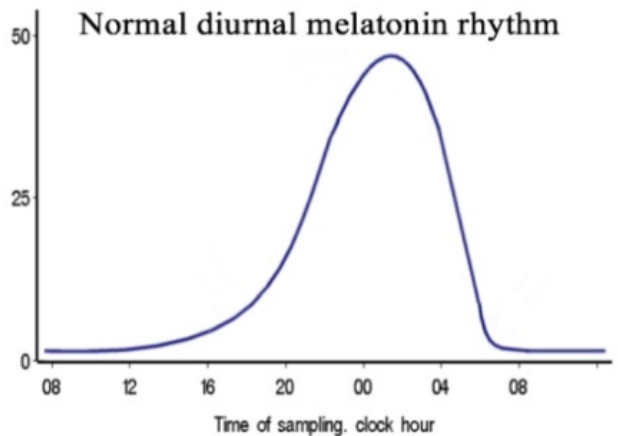

Molatonin pqimL

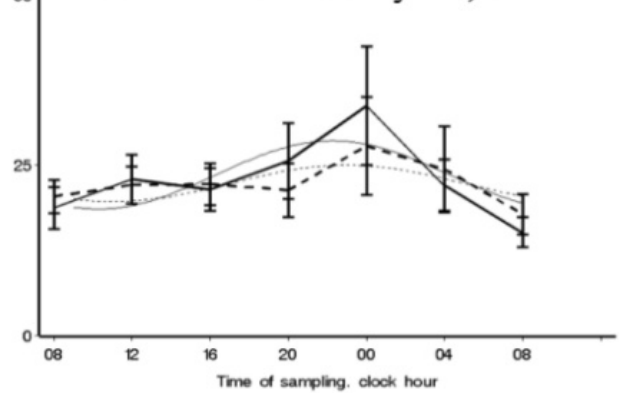

$\mathrm{D}$

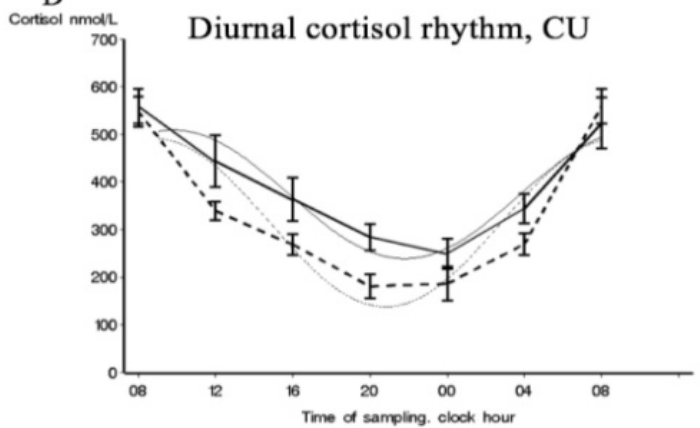

F

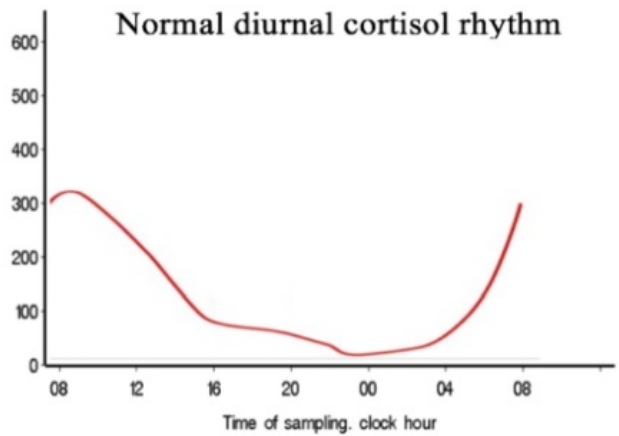

Figure 2. Patients' 24-hour blood levels of melatonin and cortisol at each time-point at inclusion and discharge. Best-fitting cosine curves (bolt and gray) and chronograms (mean and standard errors) for melatonin and cortisol at each time-point at inclusion (solid line) and at discharge (dotted line) in the intervention unit (IU) and the control unit (CU). E and $\mathrm{F}$ show visual schematic examples of normal 24-h rhythms of cortisol and melatonin.

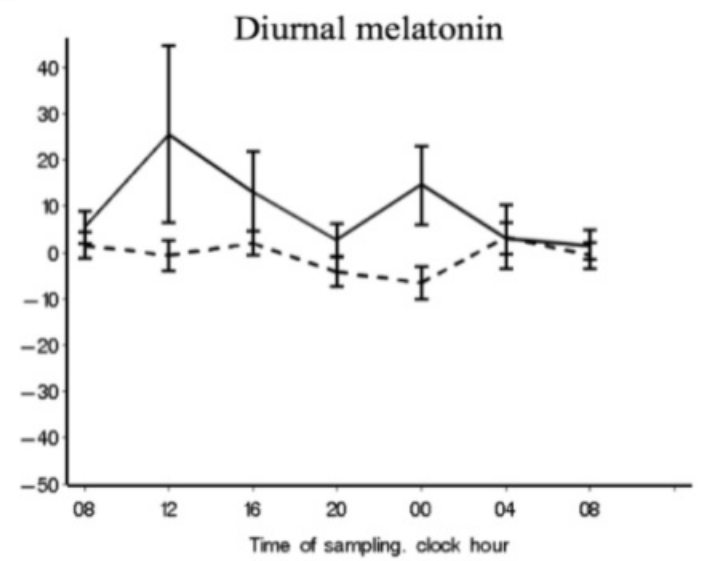

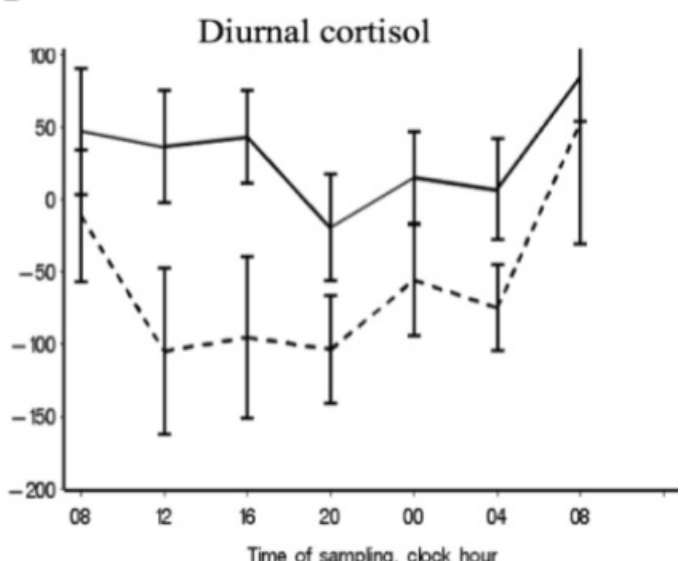

Figure 3. Chronograms for melatonin and cortisol. Chronograms of the full dataset showing the differences in the mean blood levels of melatonin (A) and cortisol (B) at inclusion and discharge in the intervention unit (solid line) and the control unit (dotted line). 
Table 4. Changes of melatonin and cortisol levels from inclusion to discharge in each unit.

\begin{tabular}{|c|c|c|c|c|}
\hline Parameter & Inclusion blood levels median (IQR) & Discharge blood levels median (IQR) & Differences in blood levels median (IQR) & $P$ value \\
\hline \multicolumn{5}{|l|}{ Mean melatonin, 24-h } \\
\hline Control Unit $(N=19)$ & $18.9(8.1$ to 27.3$)$ & 20.7 (7.9 to 23.1$)$ & $1.5(-7.0$ to 6.3$)$ & NS \\
\hline Intervention Unit $(N=23)$ & 20.3 (5.3 to 22.9$)$ & $28.9(6.0$ to 35.0$)$ & $2.9(-1.0$ to 9.9$)$ & 0.030 \\
\hline \multicolumn{5}{|l|}{ Mean cortisol, 24-h } \\
\hline Control Unit $(N=20)$ & $361.6(250.5$ to 416.6$)$ & 334.5 (188.0 to 387.8$)$ & $-59.6(-84.3$ to 33.4$)$ & NS \\
\hline Intervention Unit $(N=22)$ & $295.9(229.1$ to 316.4$)$ & $301.6(237.3$ to 434.6$)$ & $8.5(-39.9$ to 77.1$)$ & NS \\
\hline \multicolumn{5}{|l|}{ Cortisol, day ${ }^{*}$} \\
\hline Control Unit $(N=20)$ & 274.8 (169.5 to 325.8$)$ & 198.8 (76.0 to 268.2$)$ & $-59.6(-129.4$ to 13.2$)$ & 0.003 \\
\hline Intervention Unit $(N=22)$ & 209.8 (117.3 to 255.0$)$ & 204.3 (93.3 to 278.3 ) & $5.6(-68.7$ to 59.7$)$ & NS \\
\hline \multicolumn{5}{|l|}{ Cortisol, night $t^{* *}$} \\
\hline Control Unit $(N=20)$ & 444 (245.5 to 478.6$)$ & 400.1 (266.3 to 504.0$)$ & $-17.4(-76.6$ to 70.1$)$ & NS \\
\hline Intervention Unit $(N=22)$ & $364.4(259.0$ to 435.3$)$ & $401.8(255.3$ to 504.0$)$ & $28.4(-53.8$ to 107.3$)$ & NS \\
\hline
\end{tabular}

Melatonin values $(\mathrm{pg} / \mathrm{mL})$ are calculated from mean blood levels from all time-points together $(24 \mathrm{~h})$. Mean cortisol blood levels (nmol/L) were separately determined for day and night because of the preserved diurnal rhythm. The non-parametric paired test/Wilcoxon signed-rank test was used to calculate the statistical difference; therefore, median and interquartile range is given. ${ }^{*}$ Time $12-24$. "*Time $24-12$. NS $=$ Not significant.

A significant cortisol cosinor rhythm $(p<0.0001)$ was detected in both patient groups at both inclusion and discharge (Table 2, Figure 2,c,d). The CU group showed a significant amp/peak difference in cortisol values between inclusion and discharge $(p=0.005)$ which illustrate the decrease in cortisol levels between inclusion and discharge (Figure 2,d). Cosinor analysis and the calculated variance between time-points revealed that both groups showed a significant cortisol rhythm at both inclusion and discharge but that the diurnal rhythm also changes in the variance of the rhythmic pattern between inclusion and discharge at both unit (Table 3, Table S4), which 3,b also illustrate. The curves in Figure 3,b illustrate that the largest discrepancy between groups was during the first part of the day, when the $\mathrm{CU}$ group showed decreasing levels and the IU group showed stable levels.

\section{Mean levels of plasma melatonin and serum cortisol}

Table 4 summarizes the differences in melatonin and cortisol levels between inclusion and discharge for all patients.

Melatonin plasma values significantly increased from inclusion to discharge in the IU group $(n=23$; median diff, 2.9; IQR: -1.0 to 9.9; $p=0.030$ ), but not in the CU group ( $n=19$; median diff, -1.5 ; IQR: -7.0 to $6.3 ; p=0.418$ ) (Table 4). Figure 3 , a shows the melatonin delta-curve, illustrating the melatonin level changes between inclusion and discharge in the IU and not in the CU groups, and supporting a 24-hour increase.

The mean day cortisol serum levels significantly decreased from inclusion to discharge in the $\mathrm{CU}$ group ( $n=20$; median diff, -59.6 ; IQR: -129.4 to 13.2; $p$ $=0.003)$, but did not significantly change in the IU group ( $n=22$; median diff, 5.6; IQR: -68.7 to 59.7; $p=$ 0.945). During the admission time-period, cortisol night values increased in the IU group, and decreased in the CU group which is illustrated by Figure 3,b. However, these changes were not statistical significant (Table 4).
Analysis of covariance was performed to investigate cortical, striatocapsular, and large infarcts as confounding factors for the influence on melatonin and cortisol levels. Cortisol and melatonin levels were not significantly associated with these infarction types. Regression analysis revealed that lesion size was also not significantly correlated with melatonin $(n$ $=27$; Estimate, $-0.03 ; 95 \%$ CI: $-1.4,0.09 ; p=0.62$ ) or cortisol values $(n=26$; Estimate, 0.027 ; $95 \%$ CI: -0.34 , $0.88 ; p=0.38)\left(\right.$ Estimate $=$ diff. lesion size $\left.\mathrm{mm}^{3}\right)$. Regression analysis also showed that length of hospitalization was not significantly correlated with melatonin or cortisol levels.

\section{Discussion}

This study is the first to investigate the effect of a naturalistic light environment exposure on melatonin and cortisol levels in stroke patients during at least 2 weeks of hospitalization.

At the time of inclusion in our study, the stroke patients in both groups exhibited an eradicated normal diurnal pattern of melatonin, with the lack of a normal peak. At discharge, the IU group exhibited significantly increased plasma melatonin levels and a present but abnormal diurnal rhythmicity. Conversely, the CU group exhibited significant but abnormal diurnal rhythmicity at inclusion, which was absent at discharge. The absent peak levels and disrupted diurnal rhythm of melatonin in our cohort is in line with the impaired melatonin secretion and disturbed rhythmicity commonly reported after stroke.

Since melatonin is synthetized from serotonin, it is reasonable to believe that melatonin production could be affected by the known reduction/ disturbances of serotonin synthesis after stroke $[26,27]$. This could explain the absence of a melatonin secretion peak in our study. Furthermore, it has been suggested that widespread cortical lesions could affect areas projecting to the intergeniculate leaflet (IGL), potentially impairing light perception to the SCN and the pineal gland, and disrupting circadian rhythm 
regulation and melatonin secretion [6]. However, we did not find that melatonin and cortisol values were significantly correlated with lesion size, or with cortical and striatum infarcts. Notably, not all patients underwent MRI scanning; thus, the correlation was only calculated in a subgroup of patients, potentially influencing the results.

Blue light exposure during the day reportedly increases nightly melatonin secretion $[28,29]$ and prevents the melatonin suppression caused by light exposure at night [30]. This may explain the high melatonin secretion in the IU group compared to the $\mathrm{CU}$ group. The increased melatonin levels in the IU group appeared to persist over the 24-hour measurement period (Figure 3,a) despite the high exposure to the blue light spectrum at the start of the day. Although the physiological explanation is not immediately evident, it may be related to the disturbed diurnal rhythm. The $\mathrm{CU}$ group had reduced exposure to blue light during the daytime, which could make the melatonin suppression more sensitive to light [31] and inhibit melatonin secretion [32]. This might result in the $\mathrm{CU}$ group having lower melatonin levels than the IU group during the daytime, as well as at nighttime since the CU group was frequently exposed to blue light-emitting ward lights in their rooms at night. Beta-blockers have been shown to reduce the production of melatonin [33]. Beta-blockers are widely used in stroke prevention and therefore in our patient cohort. However, the distribution of beta-blockers between the two units was unequal, as there was a greater prescription at the IU (Inclusion: IU; N=12, CU; N=6. Discharge: IU; $\mathrm{N}=18, \mathrm{CU} ; \mathrm{N}=8$ ). This unequal distribution may have hypothetically decreased the melatonin production at the IU compared with the CU.

Compared to melatonin, less is understood about cortisol's response to light. We found no change in cortisol levels in the IU group, but significantly reduced cortisol levels in the $\mathrm{CU}$ group. The higher cortisol levels in the IU group compared to the $\mathrm{CU}$ group may be correlated with positive health effects, such as improved cognition, mood, and well-being [18]. However, these correlations could also be related to the light-enhanced cortical activity [34].

Unlike the melatonin rhythm, the human cortisol rhythm does not seem to be associated with day and night. However, cortisol secretion is dependent on the phase of light, particularly transition periods from dark to light and, to a lesser extent, from light to dark. The IU and CU groups showed the greatest difference in cortisol serum levels during the first part of the day period (Table 4 and Figure $3, b)$. This corresponds well with previous findings that cortisol levels increase in response to the change from dim light to bright light exposure in the morning, but not in the afternoon or night $[18,35,36]$. However, it would also be expected that bright light would not affect cortisol levels during the afternoon or nighttime, since cortisol production is usually low at those times.

Our results showed a discrepancy between the circadian rhythms of melatonin and cortisol. While the normal 24-h rhythm of melatonin secretion was eradicated, the normal 24-h rhythm of cortisol was preserved. This preserved cortisol rhythmicity is not evident for a normal preserved SCN function. Even in the absence of a functional SCN pacemaker, the adrenal gland and its own clock system can still be light-entrained by gating the sensitivity of the adrenal to ACTH via modulation of circadian corticosterone rhythms [37]. Although stroke hypothetically leads to IGL destruction, cortisol may be less sensitive to reduced IGL function and impaired serotonin levels than melatonin, due to its different approaches to light and its secondary circadian control. It remains uncertain whether this persists throughout a patient's hospitalization. It is possible that the preserved 24-h cortisol rhythm resulted from a combination of the HPA axis and the autonomic nervous system, and their activation and inhibition from the SCN.

\section{Limitations and strengths}

Patients were randomly allocated following the normal procedure for an equal distribution of patients to the two rehabilitations units (quasi-randomization). The conditions in the two rehabilitation units were equal with regards to size, form, and staff professions. The impact of daylight on the facade of the two units was not completely identical, since the angle of sun exposure differed between the two wings during all four seasons. However, measurement of the incoming sunlight revealed no significant differences between the two units [14], assuming that levels above 200 lux were required to stimulate the circadian center [38]. As illustrated in West et al. [14], there was no appreciable difference between units in daylight exposure at the window side bed across the year other than the use of curtains in the IU. There was a difference in daylight exposure between IU and CU at the bed nearest to the door, but all illuminance levels fall below the required level of 200 lux D55 equivalent light to generate a diurnal stimulation of the circadian center [38]. Thus, we do not view this difference as clinically important. Furthermore, it does not favor the IU. The intervention unit had blackout curtains that went up at 08 a.m. and down at 08 p.m. during all four seasons. It was estimated that the light significantly differed between beds during $40 \%$ of the meteorological time, over a five-hour period, during 
the peak summer season, and this difference disappeared outside the summer period. During the study period, information was collected on all bed positions, and all patients were placed near the window at the end of their stay due to the natural rotation in the units. Overall, we found no differences in bed positions between patients; thus, bed positions were excluded from the calculations. Artificial light sources at the control unit were normal indoor ceiling luminaries and a bedside lamp. The use of these light sources could not be measured due to the random use seen in a normal ward and because of the absent in manipulation of the light sources due to the control setup. The technical light description regarding the ceiling light at the control unit is described in the method description paper [14].

Blood testing could only be performed for 43 participants. The two units significantly differed with regards to smoking $(p=0.02)$, which we considered to be a random finding. NIHSS and Barthel scores significantly differed between the included and excluded participants, which were expected since the most severely impaired patients had the most difficulties participating in blood collection. At the start of the study period, saliva collection was tested as a method; however, the stroke patients showed a lack of saliva production, making this method unusable. Due to the RCT study design, all participants were equally disturbed during blood collection, for example, by waking for evening sampling.

Strengths of this study include the power of having two comparable units, and the ability to include data for all four seasons, since sunlight exposure in Denmark significantly changes throughout the year. This study was performed in a real-hospital setting; therefore, the results reflect the real-life situation in a rehabilitation hospital ward. However, this study was part of an exploratory investigational study in a relatively new scientific area. Thus, more specific studies are needed to further address the effects of naturalistic light on the levels and rhythmicity of melatonin and cortisol.

\section{Conclusions}

The present results indicate a physiologically influence of naturalistic light on melatonin and cortisol levels in patients hospitalized more than 2 weeks. There exists a need for clinical trials in circadian rhythm research with patients in a real-world clinical setting, and our study addresses that need. These findings demonstrate a rationale for further investigations on the exact implications of the observed circadian rhythm alterations, and to examine the long-term effects of the circadian light intervention.

\section{Supplementary Material}

Supplementary tables.

http://www.medsci.org/v16p0125s1.pdf

\section{Acknowledgments}

We are deeply grateful to the stroke patients for their participation in this study. We thank service manager Svend Morten Christiansson and architect Maj Lis Brunsgård Seligmann from the Service Center, Rigshospitalet Glostrup, for their interest in naturalistic light, and for making it possible to install naturalistic lighting throughout an entire hospital ward. We thank the company ChromaViso especially Master in optical engineering Torben Skov Hansen for always being available for technical questions and assistance regarding the light set-up and light description. We thank Nina Vindegaard Grønberg, MD, who was a great help in collecting data during periods of high work pressure. Finally, we are grateful to the health staff of the entire stroke department, Rigshospitalet Glostrup, for their engagement and professionalism as they provided support and logistical assistance during the project period. The last gratitude goes to The Market Development Foundation Denmark for financing the project.

\section{Competing Interests}

The authors have declared that no competing interest exists.

\section{References}

1. Thapan K, Arendt J, Skene DJ. An action spectrum for melatonin suppression: evidence for a novel non-rod, non-cone photoreceptor system in humans. J. Physiol. (Lond.) 2001;535:261-7.

2. Morin LP. Serotonin and the regulation of mammalian circadian rhythmicity. Ann. Med. 1999;31:12-33.

3. Shanahan TL, Kronauer RE, Duffy JF, Williams GH, Czeisler CA. Melatonin Rhythm Observed throughout a Three-Cycle Bright-Light Stimulus Designed to Reset the Human Circadian Pacemaker. J. Biol. Rhythms 1999;14:237-53.

4. Frisk U, Olsson J, Nylén P, Hahn RG. Low melatonin excretion during mechanical ventilation in the intensive care unit. Clin. Sci. 2004;107:47-53.

5. Guaraldi P, Sancisi E, La Morgia C, Calandra-Buonaura G, Carelli V, Cameli $\mathrm{O}$, et al. Nocturnal melatonin regulation in post-traumatic vegetative state: A possible role for melatonin supplementation? Chronobiol Int 2014;31:741-5.

6. Beloosesky Y, Grinblat J, Laudon M, Grosman B, Streifler JY, Zisapel N. Melatonin rhythms in stroke patients. Neuroscience Letters 2002;319:103-6.

7. Fiorina P, Lattuada G, Silvestrini C, Ponari O, Dall'Aglio P. Disruption of nocturnal melatonin rhythm and immunological involvement in ischaemic stroke patients. Scand. J. Immunol. 1999;50:228-31.

8. Atanassova PA, Terzieva DD, Dimitrov BD. Impaired Nocturnal Melatonin in Acute Phase of Ischaemic Stroke: Cross-Sectional Matched Case-Control Analysis. Journal of Neuroendocrinology 2009;21:657-63.

9. Zuurbier LA, Ikram MA, Luik AI, Hofman A, Van Someren EJW, Vernooij MW, et al. Cerebral small vessel disease is related to disturbed 24-h activity rhythms: a population-based study. Eur J Neurol 2015;22:1482-7.

10. Reddy AB, Maywood ES, Karp NA, King VM, Inoue Y, Gonzalez FJ, et al. Glucocorticoid signaling synchronizes the liver circadian transcriptome. Hepatology 2007;45:1478-88.

11. Scheer FAJL, Van Paassen B, Van Montfrans GA, Fliers E, Van Someren EJW, Van Heerikhuize JJ, et al. Human basal cortisol levels are increased in hospital compared to home setting. Neuroscience Letters 2002;333:79-82. 
12. Gögenur I, Ocak U, Altunpinar Ö, Middleton B, Skene DJ, Rosenberg J. Disturbances in Melatonin, Cortisol and Core Body Temperature Rhythms after Major Surgery. World J. Surg. 2006;31:290-8.

13. Riutta A, Ylitalo P, Kaukinen S. Diurnal variation of melatonin and cortisol is maintained in non-septic intensive care patients. Intensive Care Med 2009;35:1720-7.

14. West A, Jennum P, Simonsen SA, Sander B, Pavlova M, Iversen HK. Impact of naturalistic lighting on hospitalized stroke patients in a rehabilitation unit: Design and measurement. Chronobiol Int 2017;34:687-97.

15. Dijk D-J, Duffy JF, Silva EJ, Shanahan TL, Boivin DB, Czeisler CA. Amplitude Reduction and Phase Shifts of Melatonin, Cortisol and Other Circadian Rhythms after a Gradual Advance of Sleep and Light Exposure in Humans. PLoS ONE 2012;7:e30037.

16. Wright HR, Lack LC, Kennaway DJ. Differential effects of light wavelength in phase advancing the melatonin rhythm. J Pineal Res 2004;36:140-4.

17. Figueiro MG, Rea MS. The Effects of Red and Blue Lights on Circadian Variations in Cortisol, Alpha Amylase, and Melatonin. International Journal of Endocrinology 2010;2010:1-9.

18. Gabel V, Maire M, Reichert CF, Chellappa SL, Schmidt C, Hommes V, et al. Effects of Artificial Dawn and Morning Blue Light on Daytime Cognitive Performance, Well-being, Cortisol and Melatonin Levels. Chronobiol Int 2013;30:988-97.

19. Lockley SW, Brainard GC, Czeisler CA. High Sensitivity of the Human Circadian Melatonin Rhythm to Resetting by Short Wavelength Light. J. Clin. Endocrinol. Metab. 2003;88:4502-2.

20. Perras B, Meier M, Dodt C. Light and darkness fail to regulate melatonin release in critically ill humans. Intensive Care Med 2007;33:1954-8.

21. De Rui M, Middleton B, Sticca A, Gatta A, Amodio P, Skene DJ, et al. Sleep and Circadian Rhythms in Hospitalized Patients with Decompensated Cirrhosis: Effect of Light Therapy. Neurochem Res 2014;40:284-92.

22. Lucas RJ, Peirson SN, Berson DM, Brown TM, Cooper HM, Czeisler CA, et al. Measuring and using light in the melanopsin age. Trends in Neurosciences 2014;37:1-9.

23. Horne JA, Ostberg O. A self-assessment questionnaire to determine morningness-eveningness in human circadian rhythms. Int J Chronobiol 1976;4:97-110

24. Nelson W, Tong YL, Lee JK, Halberg F. Methods for cosinor-rhythmometry. Chronobiologia 1979;6:305-23.

25. Cornelissen G. Cosinor-based rhythmometry. Theoretical Biology and Medical Modelling 2014;11:1-24.

26. Véra P, Zilbovicius M, Chabriat H, Amarenco P, Kerdraon J, Ménard JF, et al. Post-stroke changes in cortical 5-HT2 serotonergic receptors. J. Nucl. Med. 1996;37:1976-81.

27. Martín A, Szczupak B, Gómez-Vallejo V, Plaza S, Padró D, Cano A, et al. PET Imaging of Serotoninergic Neurotransmission with [ 11C]DASB and [ 18F]altanserin after Focal Cerebral Ischemia in Rats. Journal of Cerebral Blood Flow \& Metabolism 2013;33:1967-75.

28. Hashimoto $S$, Kohsaka $M$, Nakamura $K$, Honma $H$, Honma $S$, Honma $K$. Midday exposure to bright light changes the circadian organization of plasma melatonin rhythm in humans. Neuroscience Letters 1997;221:89-92.

29. Park S-J, Tokura H. Bright Light Exposure During the Daytime Affects Circadian Rhythms of Urinary Melatonin and Salivary Immunoglobulin A. Chronobiol Int 2009;16:359-71.

30. Kozaki T, Kubokawa A, Taketomi R, Hatae K. Light-induced melatonin suppression at night after exposure to different wavelength composition of morning light. Neuroscience Letters 2016;616:1-4.

31. Giménez MC, Beersma DGM, Bollen P, van der Linden ML, Gordijn MCM. Effects of a chronic reduction of short-wavelength light input on melatonin and sleep patterns in humans: Evidence for adaptation. Chronobiol Int 2014;31:690-7.

32. Mishima K, Okawa M, Shimizu T, Hishikawa Y. Diminished melatonin secretion in the elderly caused by insufficient environmental illumination. J. Clin. Endocrinol. Metab. 2001;86:129-34.

33. Stoschitzky K, Sakotnik A, Lercher P, Zweiker R, Maier R, Liebmann P, et al. Influence of beta-blockers on melatonin release. Eur. J. Clin. Pharmacol. 1999;55:111-5.

34. Perrin F, Peigneux P, Fuchs S, Verhaeghe S, Laureys S, Middleton B, et al. Nonvisual Responses to Light Exposure in the Human Brain during the Circadian Night. Current Biology 2004;14:1842-6.

35. Scheer FA, Buijs RM. Light affects morning salivary cortisol in humans. J. Clin. Endocrinol. Metab. 1999;84:3395-8.

36. Leproult R, Colecchia EF, L'Hermite-Balériaux M, Van Cauter E. Transition from dim to bright light in the morning induces an immediate elevation of cortisol levels. J. Clin. Endocrinol. Metab. 2001;86:151-7.

37. Oster H, Damerow S, Kiessling S, Jakubcakova V, Abraham D, Tian J, et al. The circadian rhythm of glucocorticoids is regulated by a gating mechanism residing in the adrenal cortical clock. Cell Metabolism 2006;4:163-73.

38. Andersen M, Mardaljevic J, Lockley S. A framework for predicting the non-visual effects of daylight - Part I: photobiology- based model. Lighting Research and Technology 2012;44:37-53. 